\title{
Open Median Nerve Release Using Double Mini Skin Incision in Patients With Carpal Tunnel Syndrome: Technique and Clinical Results
}

\author{
Cengiz ÇOKLUK, Alparslan ŞENEL, Ömer İYIGÜN, Fahrettin ÇELIK, \\ and Cemil RAKUNT
}

Department of Neurosurgery, Medical Faculty, Ondokuzmayıs University, Samsun, Turkey

\begin{abstract}
Patients who undergo open carpal tunnel surgery frequently complain of the postoperative cosmetic appearance at the site of the incision on the palm. This problem occurs as a result of excessive scar formation, and the long incision. A double mini skin incision, each $1 \mathrm{~cm}$ long, was used in the surgical treatment of carpal tunnel syndrome. The transverse carpal ligament was easily sectioned. Postoperatively healing was good with no excessive scar formation.
\end{abstract}

Key words: double mini skin incision, open median nerve release, carpal tunnel syndrome

\section{Introduction}

Carpal tunnel syndrome is one of the most common entrapment syndromes encountered in the practice of peripheral nerve surgery, and results from manipulations requiring repetitive wrist motion which promote median nerve entrapment at the wrist. ${ }^{1,2)}$ Approximately $1 \%$ of population is affected by carpal tunnel syndrome.1,2) History, physical examination, electromyography, and nerve conduction velocity measurements are essential for the diagnosis. ${ }^{14)}$ Surgical division of the transverse carpal ligament is the generally accepted surgical technique for decompression of the entrapped median nerve..$^{2,3,5)}$

Unesthetic wound healing and excessive scar formation on the palm are recognized as serious cosmetic problems influencing the quality of life in patients undergoing open median nerve release. These types of cosmetic problems can affect patient satisfaction, even after successful treatment of the pathology. Various limited skin incisions are regarded as minimally invasive and effective for preventing excessive scar formation and achieving better cosmetic results. ${ }^{9,12,13)}$

Here we describe the surgical technique of median nerve release using double mini skin incisions, and evaluate wound healing, cosmetic results, and scar formation on the palm.

Received January 6, 2003; Accepted April 10, 2003

\section{Surgical Technique and Results}

Before surgery, the forearm, wrist, and hand were cleaned with povidone iodine solution (Isosol $1000 \mathrm{ml}$; Merkez Laboratuarı, Istanbul, Turkey) to provide sterile surgical conditions. The surgical incision line was drawn with a sterile surgical pen. The surrounding area was covered with sterile cotton compress to isolate the hand. The operation was performed under local anesthesia induced with $2 \%$ prilocaine hydrochloride (Citanest $20 \mathrm{ml}$; Flacon Astra Zeneka, Istanbul, Turkey). No tourniquet was used in any patient. An operative loupe for magnification of the field was used in all patients.

The imaginary line running from the distal wrist crease to the crossing point of two perpendicular lines, extending to the inferior edge of the extended thumb and to the line between third and fourth digits, was divided into three equal parts. The proximal and distal skin parts were opened, but the middle segment was kept intact (Fig. 1A). The proximal incision was started from just distal to the wrist flexion crease and extended for $1 \mathrm{~cm}$ at 1-2 $\mathrm{mm}$ on the ulnar side of the thenar crease. Two mini skin retractors were used to retract the incisioned skin (Fig. 1B).

The transverse carpal ligament was cut off from the inferior edge to the superior edge, between the ulnar neurovascular bundle and the lateral aspect of the median nerve. Extension of the wrist can help in the identification of the transverse carpal ligament 


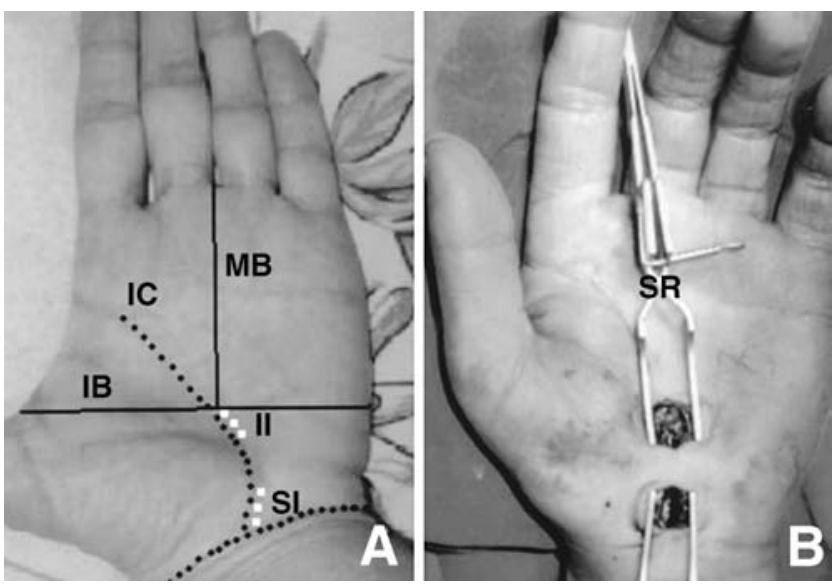

Fig. 1 Photographs illustrating the double mini skin incision technique. A: The imaginary lines of the double mini skin incision. IB: inferior border, the line extends along the inferior edge of the extended thumb; IC: interthenar crease; II: inferior incision; MB: midline border, the line between the third and fourth digits; SI: superior incision. B: Intraoperative photograph showing the two small incisions retracted with small self-retaining retractors (SR).

and the median nerve segment under the unincisioned skin segment. The ligament was cut off as far as the inferior edge of the proximal incision. The proximal part of the ligament was sectioned through the proximal skin incision. The sectioning of the ligament was checked along the carpal tunnel, and the median nerve was inspected. The skin was sutured with $4 / 0$ vicryl sutures without subcutaneous suture. The skin sutures were removed 10 days after the operation.

The limited double skin incision technique was used in 24 female patients (mean age $47 \pm 6$ years). The mean follow-up period was $24 \pm 2$ months. There was no case of reoperation or skin infection. No palmar cutaneous branch injury was detected. The cosmetic appearance was better compared to our patients who underwent the standard incision during the same period. The wound healing was better, and no excessive scar formation leading to the cosmetic disorganization was detected. No patients reported any discomfort caused by the scar tissue on the palm during shaking hands. Our postoperative observations revealed that patients who were treated with the double mini skin incision had better and faster wound healing and an easier postoperative course than those with standard incisions.

\section{Discussion}

Open transverse carpal ligament transection for median nerve release can be performed through various skin incisions of the palm..$^{2,8,9,13,16,17)}$ The standard skin incision for carpal tunnel surgery runs from the wrist flexion crease, and extends curvilinearly to a point in line with the distal border of the fully extended thumb. ${ }^{11)}$ In this technique, the incision is generally placed 2 to $3 \mathrm{~mm}$ medial to the volar crease, in line with the long axis of the ring finger. This incision is commonly known as the standard skin incision. Recently, other minimally invasive skin incisions had been described for surgical intervention..$^{9,12,13,17)}$ The primary goal of limited skin incisions is to achieve better cosmetic results, lesser scar formation and decreased postoperative pain on the surface of palm..$^{2,8,9,12,13,16,17)}$ As many as $24 \%$ of postoperative problems are related to skin incision. ${ }^{1)}$ Hypertrophic and/or sensitive scar formation, and tenderness are the most common sequelae. ${ }^{1,2)}$

Our double mini skin incision technique uses two separate incisions located along the line of the standard incision. The medium segment remained intact. To identify the segments of the double mini skin incisions, the imaginary line of the standard incision should be divided into three equal segments. The proximal and distal segments are incisioned. Subcutaneous dissection at the proximal and distal incisions was easy and retraction exposed the superior and inferior parts of transverse carpal ligament. The middle part of the ligament was easily sectioned under direct visualization through the inferior incision. In some cases, gentle extension of the wrist is necessary for better visualization of the ligament under the unincisioned skin. A surgical loupe is necessary for magnification. The operating microscope also can be used. The palmar cutaneous branch and thenar motor branch of the median nerve, and the superficial palmar arch can be easily preserved with this technique. The distal portion of the transverse carpal ligament is divided under direct vision through the inferior portion of the double incision. The proximal part of the incision provides wide exposure to decompress the median nerve at the site of the wrist joint. Our experience revealed that hypertrophic dolorous skin scarring may occur at the wrist fold in the early postoperative follow-up period (first 3 months), but then gradually decreases.

Endoscopic carpal tunnel surgery is a new surgical technique which has been used to decompress the median nerve in patients with carpal tunnel syndrome for the past decade., ${ }^{4,7,15)}$ No meaningful 
differences have been reported between the open and endoscopic techniques in the aspects of operating time and cost of surgery. ${ }^{6,15)}$ The advantage of the endoscopic technique is reduced scar formation on the palmar surface of the hand. ${ }^{7,15)}$ On the other hand, the endoscopic technique has some disadvantages such as high complication and recurrence rates. ${ }^{4,7,10)}$

The human hands are not only a biomechanical organ but also a tactile sensory apparatus of the body. Peoples whose jobs require repetitive wrist motion are more affected by carpal tunnel syndrome ${ }^{1,2)}$ but the patients want to return to their jobs as soon as possible after the operation with normal work capacity including cosmetic appearance. Therefore, normal hand function without deficits, resolution of the symptoms related to median nerve entrapment, and a good postoperative cosmetic appearance are all extremely important in the assessment of surgical success. Our clinical observations revealed the patients who were treated with the double mini skin incision had a good postoperative cosmetic appearance with normal tactile and mechanical hand functions.

The postoperative cosmetic appearance of the palm is an important issue related to skin incision. Long incisions are usually associated with hypertrophic scar formation leading to difficulties with the hand functions and esthetic dissatisfaction. We suggest that the double mini skin incision be selected for the open surgical treatment of carpal tunnel syndrome.

\section{References}

1) Abdullah AF, Wolber PH, Ditto EW: Sequelae of carpal tunnel surgery: rationale for the design of a surgical approach. Neurosurgery 37: 931-936, 1995

2) Biyani A, Downes EM: An open twin incision technique of carpal tunnel decompression with reduced incidence of scar tenderness. J Hand Surg [Br] 18: 331-334, 1993

3) Bromley GS: Minimal-incision open carpal tunnel decompression. J Hand Surg [Am] 19: 119-120, 1994

4) Concannon MJ, Brownfield ML, Puckett CL: The incidence of recurrence after endoscopic carpal tunnel release. Plast Reconstr Surg 105: 1662-1665, 2000

5) Delaere O, Bouffioux N, Hoang P: Endoscopic treat- ment of the carpal tunnel syndrome: review of the recent literature. Acta Chir Belg 100: 54-57, 2000

6) Ferdinand RD, MacLean JG: Endoscopic versus open carpal tunnel release in bilateral carpal tunnel syndrome. A prospective, randomised, blinded assessment. J Bone Joint Surg Br 84: 375-379, 2002

7) Filippi R, Reisch R, El-Shki D, Grunert P: Uniportal endoscopic surgery of carpal tunnel syndrome: technique and clinical results. Minim Invasive Neurosurg 45: 78-83, 2002

8) Lee WP, Strickland JW: Safe carpal tunnel release via a limited palmar incision. Plast Reconstr Surg 101: 418-426, 1998

9) Nathan PA: Carpal tunnel release using minimally invasive technique. Plast Reconstr Surg 99: 1195-1196, 1997

10) Palmer AK, Toivonen DA: Complications of endoscopic and open carpal tunnel release. J Hand Surg [Am] 24: 561-565, 1999

11) Rengachary S: Entrapment neuropathies, in Wilkins R, Rengachary S (eds): Neurosurgery. New York, McGraw-Hill Book, 1985, pp 1771-1776

12) Schmidt W, Gruber AA, Hammer R: [Results of different incisions in treatment of carpal tunnel syndrome]. Handchir Mikrochir Plast Chir 32: 67-69, 2000 (Ger, with Eng abstract)

13) Serra JM, Benito JR, Monner J: Carpal tunnel release with short incision. Plast Reconstr Surg 99: 129-135, 1997

14) Shapiro S: Microsurgical carpal tunnel release. Neurosurgery 37: 66-70, 1995

15) Trumble TE, Diao E, Abrams RA, Gilbert-Anderson MM: Single-portal endoscopic carpal tunnel release compared with open release: a prospective, randomized trial. J Bone Joint Surg Am 84-A: 1107-1115, 2002

16) Wilsom KM: Double incision open technique for carpal tunnel release: an alternative to endoscopic release. J Hand Surg [Am] 19: 907-912, 1994

17) Zimmerli W: [Double incision for operation of carpal tunnel syndrome -14 years experience]. Helv Chir Acta 58: 395-400, 1992 (Ger, with Eng abstract)

Address reprint requests to: C. Çokluk, M.D., Department of Neurosurgery, Medical Faculty, Ondokuzmayıs University, 55139 Samsun, Turkey.

e-mail: cengizcokluk@yahoo.com

Commentary on this paper appears on the next page. 


\section{Commentary}

Satisfactory results with few complications have been achieved in the past with many techniques of surgery for carpal tunnel syndrome. Considering that section of the flexor retinaculum can be performed through various skin incisions, and postoperative problems, if present, are frequently related to excessive or sensitive scar formation, it appears preferable to use more limited skin incisions instead of the relatively extensive standard incision. The favorable clinical results obtained by the authors in 24 patients with their limited double skin incision technique clearly support this minimally invasive approach. In spite of the narrow surgical field, the authors were obviously able to avoid recurrence due to insufficient median nerve release, unesthetic wound healing, scar tenderness, and injury of the cutaneous palmar and thenar motor branches of the median nerve. Personally, I have not used such a technique so far, but after reading this article, I am convinced of its benefits in the surgical treatment of this common focal neuropathy.

Helmut BERTALANFFy, M.D. Department of Neurosurgery Philipps University Hospital Marburg, Germany 